\section{PARENTS ATTITUDE TOWARD INFANT SAFETY DURING AIR TRAVEL}

\author{
M. Bahari ${ }^{1}$, N. Prunty ${ }^{1}$, E. Molloy ${ }^{1,2}$ \\ ${ }^{1}$ Neonatology, National Maternity Hospital, \\ ${ }^{2}$ Paediatrics, Royal College of Surgeons of Ireland, \\ Dublin, Ireland
}

Aims: There is a deficiency in global air travel safety provision for children especially in the under 2-yearold age group. The Federal Aviation Authority and American Academy of Paediatrics encourage the use of Child Restraint System (CRS), however it is not compulsory. There are concerns, if CRS use becomes mandatory, air travel costs will increase.

Method: Cross sectional quantitative study, between $22^{\text {nd }}$ February to $2^{\text {nd }}$ March 2010. Setting: Baby clinic in National Maternity Hospital. Sample: Parents attending baby clinic and agreed to participate. Study tool: Pilot tested, self filled questionnaire.

Results: 60 Questionnaires were distributed, 52 returned the questionnaire $(R R=86 \%)$. All respondents were females. Only $11.5 \%$ heard about CRS before but no one had ever used it before. $84 \%$ think CRS is important for infant safety and $92 \%$ are willing to purchase a separate seat for CRS. All participants believe airlines should provide CRS as basic requirement. $66 \%$ of participants are willing to pay up to $50 \%$ of the cost of a separate seat for the use of CRS. Up to $90 \%$ of participants say they had never given instruction specific to their infant safety during their air travel experience.

Conclusion: More effort is required to increase the knowledge among parents regarding infant safety during air travel. We suggest that airlines provide:

(1) standardized information on CRS use during air travel,

(2) financial incentives (discount fares) when using a CRS and

(3) an approved age-appropriate airline-suitable CRS at the departure point

\section{SHOULD MY CHILD HAVE A PET?}

M. Warren, C. Fitzgerald, J. Nolan, A. Murphy, E. Roche, J. Meehan, H. Hoey

Department of Paediatrics, Trinity College Dublin, Dublin, Ireland

Background: The keeping of pets is a common practice both nationally and internationally. Many children are exposed to pets from a very young age. Evidence in relation to their role in childhood morbidity is ambiguous. Little Irish data exists on pet demographics and the epidemiology of pet-related illnesses in children.

Aims: Our aims were to research pet demographics in Ireland, to explore the relationship between pets and childhood illnesses and to learn more about Irish parents' attitudes to pets and disease.

Methods: A literature review was carried out on the association between pets and disease in childhood. A random selection of 500 parents and children attending a Paediatric Hospital during February and March 2010 were interviewed in order to ascertain pet ownership status, associated problems and beliefs regarding risks and benefits.

Results: At present, conflicting evidence exists regarding the association of common household pets and atopy. Of the 500 households surveyed $49 \%$ owned a pet and $52 \%$ of these had multiple pets. Dogs and cats were the most popular pets among our study group but a wide range of animals were noted. One fifth of pet owners had experienced morbidity as a result of their pet.

Conclusion: The overall impression conveyed that Irish parents who had been pet owners in childhood were strong advocates of the psychological advantages of pets. The effort involved in caring for a pet rather than risk of illness was cited as the main deterrent by those who declined pet ownership. 